\title{
Synchrony of Spontaneous Burst Firing between Retinal Ganglion Cells Across Species
}

\author{
Jungryul Ahn ${ }^{1}$, Huu Lam Phan ${ }^{2}$, Seongkwang Cha ${ }^{1}$, Kyo-in $\mathrm{Koo}^{2}$, Yongseok Yoo ${ }^{3 *}$ \\ and Yong Sook Goo ${ }^{1 *}$ \\ ${ }^{1}$ Department of Physiology, Chungbuk National University School of Medicine, Cheongju 28644, \\ ${ }^{2}$ Department of Biomedical Engineering, University of Ulsan, Ulsan 44610, \\ ${ }^{3}$ Department of Electronics Engineering, Incheon National University, Incheon 22012, Korea
}

\begin{abstract}
Neurons communicate with other neurons in response to environmental changes. Their goal is to transmit information to their targets reliably. A burst, which consists of multiple spikes within a short time interval, plays an essential role in enhancing the reliability of information transmission through synapses. In the visual system, retinal ganglion cells (RGCs), the output neurons of the retina, show bursting activity and transmit retinal information to the lateral geniculate neuron of the thalamus. In this study, to extend our interest to the population level, the burstings of multiple RGCs were simultaneously recorded using a multi-channel recording system. As the first step in network analysis, we focused on investigating the pairwise burst correlation between two RGCs. Furthermore, to assess if the population bursting is preserved across species, we compared the synchronized bursting of RGCs between marmoset monkey (callithrix jacchus), one species of the new world monkeys and mouse (C57BL/6J strain). First, monkey RGCs showed a larger number of spikes within a burst, while the inter-spike interval, burst duration, and inter-burst interval were smaller compared with mouse RGCs. Monkey RGCs showed a strong burst synchronization between RGCs, whereas mouse RGCs showed no correlated burst firing. Monkey RGC pairs showed significantly higher burst synchrony and mutual information than mouse RGC pairs did. Comprehensively, through this study, we emphasize that two species have a different bursting activity of RGCs and different burst synchronization suggesting two species have distinctive retinal processing.
\end{abstract}

Key words: Information transmission, Burst correlation, Retinal ganglion cells (RGCs), Primate retina, Burst analysis

\section{INTRODUCTION}

Neurons communicate with other neurons in response to changes in the internal and external environment. Primarily, spiking neurons transmit information to other neurons in the temporal pattern of action potentials called spike train due to its all-or-none nature $[1,2]$. Their goal is to transmit information to other neu-

Submitted July 7,2020, Revised August 27,2020,

Accepted August 31,2020

* To whom correspondence should be addressed. Yongseok Yoo, TEL: 82-32-835-8453, FAX: 82-32-835-0774

e-mail:yyoo@inu.ac.kr

Yong Sook Goo, TEL: 82-43-261-2870, FAX: 82-43-272-1603

e-mail:ysgoo@chungbuk.ac.kr rons faithfully. Accordingly, it is very crucial that this spike train successfully carries a large amount of information without any loss to their targets.

Regarding the strategy of transferring information, using only one spike could be very vulnerable as it is sensitive to noise contamination and requires very accurate timing. If a spike is lost or mistimed during synaptic transmission, it can lead to a fatal loss of information. Alternatively, multiple spike trains can carry even much information without any loss, assuring the robust transmission $[3,4]$.

A burst, which consists of multiple spikes within a short time interval, plays an essential role in improving the reliability of information transmission through synapses. A single spike of a presynaptic neuron may not evoke the excitatory postsynaptic po-
Copyright (c) Experimental Neurobiology 2020. www.enjournal.org
This is an Open Access article distributed under the terms of the Creative Commons Attribution Non-Commercial License (http://creativecommons.org/licenses/by-nc/4.0) which permits unrestricted non-commercial use, distribution, and reproduction in any medium, provided the original work is properly cited. 
tential (EPSP), while a burst improves the probability of EPSP in a postsynaptic neuron [5-7]. This synaptic transmission through the burst has been widely observed in various neural systems, including Purkinje cells $[8,9]$, thalamocortical neurons [10,11], and pyramidal neurons in hippocampus [12,13]. In these neural systems, synaptic transmission through the burst can induce synaptic plasticity. For instance, in rat hippocampal pyramidal cells, the burst induced EPSP and long-term potentiation (LTP), whereas the single spike did not induce LTP [13]. This result suggests that burst firing may be associated with the modulation of the neural plasticity.

Unfortunately, our knowledge that bursts increase information transmission is limited to a single synapse. However, since most computations in the nervous system are performed by a network of synapses rather than a single synapse, so studying the burst activity at the network level will improve our understanding of the contribution of bursts to information transmission.

In the visual system, retinal ganglion cells (RGCs), the output neurons of the retina, show bursting activity in response to changes in the internal circuit or external visual environment [1416]. Mostly, this burst information of the RGCs reaches to lateral geniculate neuron (LGN) of the thalamus, which is known as the relay neuron between RGC and the primary visual cortex in the visual pathway. The bursts of RGCs augment the transmission of retinal signals to LGN and induce its synapse plasticity [17-19]. These findings emphasize the functional role of RGC bursts for information transmission in retinogeniculate communication. However, most studies are still limited to a single unit of RGC.

To extend our interest to the network level, in this study, we recorded the population bursting of RGCs using a multi-channel recording system, and the temporal correlation of bursts among RGCs was investigated through correlation analysis. As the first step in network analysis, we focused on the pairwise burst correlation between two RGCs. Furthermore, to find out if the phenomena of population bursting are preserved across species, we compared the synchronized bursting activity of RGCs between marmoset monkey and mouse. However, marmoset monkey is one species of the new world monkeys, and it is not the most representative model for primates like macaque monkeys.

This study emphasizes that two species have a different bursting activity of RGCs, and different burst synchronization. In particular, mouse RGCs hardly show synchronized bursting activity, but monkey RGCs share their bursting activity with nearby RGCs, suggesting two species have distinctive retinal processing.

\section{MATERIALS AND METHODS}

\section{Retinal preparation}

Male mice at postnatal day 56 and above (>P56) (C57BL/6J strain; The Jackson Lab., ME, USA) were used for this study ( $\mathrm{n}=6$ ). Institutional animal care committee of Chungbuk National University approved animal use protocols (approval number: CBNUA-1172-18-02). The mice were anesthetized with intramuscular injection of $30 \mathrm{mg} / \mathrm{kg}$ tiletamine-zolazepam hydroxide (Zoletil 50; Virbac, Sao Paulo, Brazil), $10 \mathrm{mg} / \mathrm{kg}$ zylazine hydrochloride (Rumpun; Bayer Korea, Seoul, South Korea), and 5000 IU heparine sodium (Heparin; JW Pharmaceutical Corp., Seoul, South Korea). Monkey eyeballs were obtained from marmoset monkeys (callithrix jacchus, $n=4$ ) used by the Laboratory Animal Center of the OSONG KBIO HEALTH, following institutional guidelines for the care and use of animals. In the process of transportation of monkey eyeballs after enucleation, $\mathrm{CO}_{2}$-independent medium, well known as transport buffer, was used to avoid tissue damage caused by $\mathrm{pH}$ changes in the hypoxic buffer $\left(\mathrm{CO}_{2}\right.$-Independent Medium; Thermo Fisher Scientific, MA, USA). Four retinas at postnatal year two and higher were used. The detailed procedures for the preparation of the ex-vivo retinal patch were described in previous studies [20,21]. Briefly, the eyeball was enucleated, and then the retina was isolated from the sclera and the retinal pigment epithelium (RPE) and cut into a $2 \times 2 \mathrm{~mm}^{2}$ patch. The retinal patch was prepared under illumination of $4.3 \mathrm{nW} / \mathrm{cm}^{2}$ in an artificial cerebrospinal fluid (ACSF) solution (124 mM NaCl, $10 \mathrm{mM} \mathrm{Glu-}$ cose, $1.15 \mathrm{mM} \mathrm{KH}_{2} \mathrm{PO}_{4}, 25 \mathrm{mM} \mathrm{NaHCO}_{3}, 1.15 \mathrm{mM} \mathrm{MgSO}_{4}, 2.5$ $\mathrm{mM} \mathrm{CaCl}$, and $5 \mathrm{mM} \mathrm{KCl}$ ) bubbled with $95 \% \mathrm{O}_{2}$ and $5 \% \mathrm{CO}_{2}$ to maintain a $\mathrm{pH}$ of $7.3 \sim 7.4$ and a temperature of $32^{\circ} \mathrm{C}$. The isolated retina was mounted the retinal ganglion cell (RGC) layer down on a planar multi-electrode array (MEA), and it was continuously perfused with oxygenated solution (flow rate: $1 \sim 3 \mathrm{ml} / \mathrm{min}$ ) during the experiment.

\section{Multi-electrode recording system and signal processing}

The data acquisition system (MEA60 system; Multichannel Systems GmbH, Reutlingen, Germany) included a planar 64-channel perforated MEA (60pMEA200/30iR), an amplifier (MEA1060), temperature control units (TC01), data acquisition hardware ( $\mathrm{Mc}$. Card) and software (Mc_Rack). The MEA contained 64 circular electrodes in an $8 \times 8$ grid layout with electrode diameters of $30 \mu \mathrm{m}$ and inter-electrode distances of $200 \mu \mathrm{m}$ (Fig. 1A). The electrodes were coated with porous titanium nitride (TiN) and were embedded in a perforated polyimide foil, which allows sufficient oxygen and nutrient supply to the retina [22]. Multi-electrode recordings of the retinal activity were obtained from 59 electrodes except for 


\section{A. Recording electrode: Multi-electrode array (MEA)}

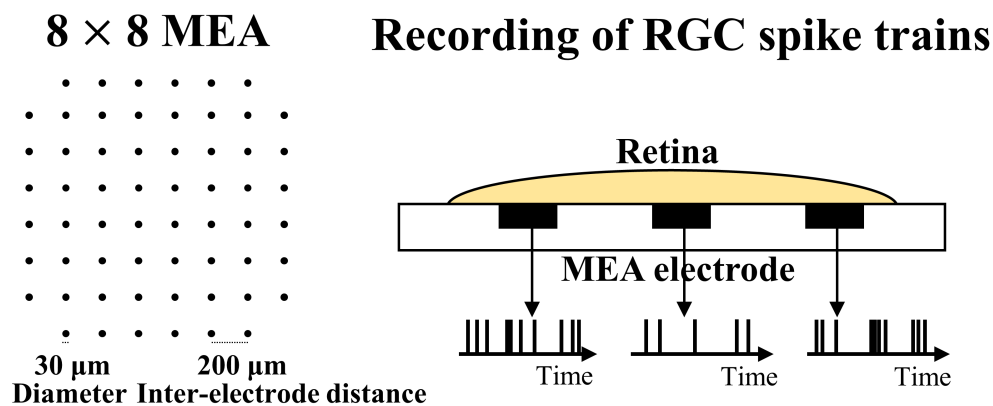

\section{B. Burst analysis}

\section{Each spike train of two RGCs}
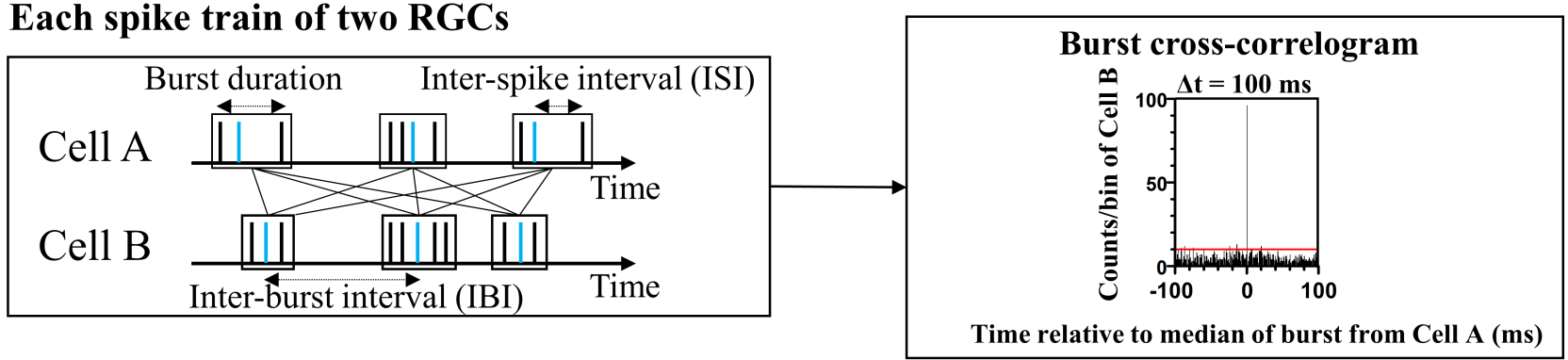

\section{Receptive field estimation \\ Random-checkerboard stimulus}

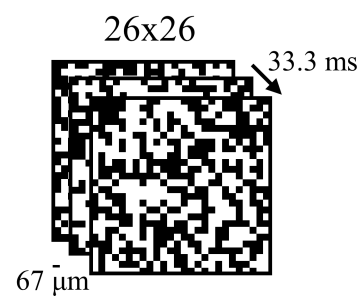

\section{RF fitting}

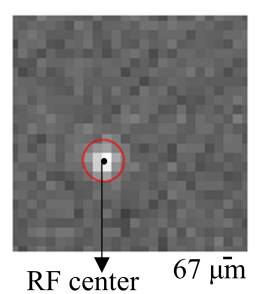

Fig. 1. Multi-channel recording system and burst analysis. (A) Configuration of a multi-electrode array (MEA) and recording of RGC spike trains. (B) Burst analysis parameters and burst cross-correlogram. Blue lines indicate the median time of each burst. The red lines indicate the significance level represented by the $99 \%$ confidence limit of the time histogram. (C) Random-checkerboard stimulation and receptive field (RF) estimation of RGC from the spatial map of spike-triggered average (STA).

one reference electrode and four inactive electrodes, with a bandwidth ranging from 1 to $3,000 \mathrm{~Hz}$ at a gain of 1,200. The data sampling rate was $25 \mathrm{kHz}$ per electrode. From the raw waveform of retinal recording, RGC spikes were isolated using a $100 \mathrm{~Hz}$ highpass filter. The threshold for spike detection was set to four times the standard deviation of the background noise. The recording data were processed with spike sorting software (Offline Sorter ${ }^{\mathrm{TM}}$; Plexon Inc., TX, USA) to separate multiunit activities containing different spike waveforms into individual cell units using principal component analysis [23]. Data analysis was processed utilizing a commercial analyzing software (NeuroExplorer ${ }^{\mathrm{TM}}$; Nex Technolo- gies, CO, USA) and a custom-made Matlab ${ }^{\mathrm{TM}}$ (MathWorks, MA, USA) code.

\section{Burst analysis}

For burst analysis, we identified the burst quantitatively using the Poisson surprise algorithm [24, 25]. First, the mean of inter-spike interval (ISI) of the whole spike train, $\partial$, was calculated. Then, under the Poisson assumption, the mean spike rate, $\lambda$, of a spike train was set to $1 / \partial$. Next, we identified three consecutive spikes with ISIs less than $\partial / 2$ as the initial burst.

Starting from the initial burst of three spikes, the following spike 
is included in the burst if the probability of the null hypothesis (independent spikes) decreases by adding the spike of question. Specifically, the null hypothesis is that $k$ consecutive spikes independently occur during the corresponding time interval of length $t$, the probability of which is given by the Poisson distribution:

\section{(Eq. 1)}

$$
\begin{aligned}
P_{k, t} & =\operatorname{Prob}(\text { observing } k \text { independent spikes during interval of length } t) \\
& =e^{-(\lambda t)} \times \frac{(\lambda t)^{k}}{k !}
\end{aligned}
$$

Thus, $P_{k, t}$ and $P_{k+1, t+t t}$ is compared, where $k$ and $t$ are the spike count and duration of the current burst, and $\Delta t$ is the time to the next spike of question. This incremental update is repeated until $P_{k, t}$ increases.

When the probability of $\mathrm{k}^{\text {th }}$ consecutive spikes $\left(P_{k, t+t t}\right)$ is less than $P_{3, t}$ of initial three consecutive spikes $\left(P_{k, t+\Delta t}<P_{3, t}\right)$, we included the $\mathrm{k}^{\text {th }}$ consecutive spikes into the burst. The spike count, $k$, was computed for the spike train in a time bin, $t$, where $t$ is the time interval between the first and last spikes of three spikes under investigation.

Finally, any bursts with $P_{k, t+\Delta t}$ less than $P_{3, t}$ were identified. The start of the burst was defined as the time of the first spike. The end of the burst was defined as the time of the last spike. Then, we computed five burst parameters such as burst duration, spike counts within a burst, ISI in a burst, burst frequency, and interburst interval (IBI) for each neuron (Fig. 1B). These were computed by averaging all values over the entire recording for analyzed neurons.

\section{Burst-correlation analysis}

The burst-correlation of two simultaneously recorded burst trains between RGCs can be quantified using the cross-correlogram, which displays the amount of synchronized burst firing between two cells as a function of the difference of the median time of each burst. First, we binned burst trains from RGC pairs to generate a binary burst count for each RGC as a function of time. Next, to quantify the strength of a correlation between two RGCs, we used the burst synchrony index (BSI). It is defined as the ratio between the probability of synchronized burst firing (i.e., two cells fire together during a time bin) and the probability expected from statistically independent firing [26]. The BSI was calculated using the following equation:

$$
B S I=\log _{2} \frac{P(A=1, B=1)}{P(A=1) P(B=1)^{\prime}}
$$

where 1 denotes the occurrence of a burst. The BSI value of two independent burst trains is zero, and a greater BSI value indicates higher synchrnony.

The time interval for calculating probabilities in Eq. 2 was chosen to be $100 \mathrm{~ms}$, considering two components (the deviation of median times for each burst and IBI values). For burst analysis, average IBI values were around $200 \mathrm{~ms}$ from both two species. Thus, the time interval should be shorter than $200 \mathrm{~ms}$. The BSI was calculated and plotted for the inter-electrode distances ranging from the nearest distance of $200 \mu \mathrm{m}$ to a maximum of $1,400 \mu \mathrm{m}$ by step of $200 \mu \mathrm{m}$.

\section{Mutual information analysis}

We measured mutual information (MI) of bursting of two RGCs in bits, which quantifies the amount of information obtained about the burst of an RGC through observation of the burst of the other RGC. In general, the MI of two variables $X$ and $Y$ determines how different the joint distribution of a pair $(X, Y)$ is from the product of the individual distributions of $X$ and $Y$. The MI of two joint random variables $X$ and $Y$ is calculated as the following equation:

(Eq. 3)

$$
I(Y ; X)=\sum_{x \in X} \sum_{y \in Y} p(x, y) \log _{2}\left(\frac{p(x, y)}{p(x) p(y)}\right)
$$

where $p(x, y)$ is the joint probability of $x$ and $y$, and $p(x)$ and $p(y)$ are the individual probabilities of $x$ and $y$, respectively. Using Eq. 3 for burst states ( 0 and 1 for absence and presence of a burst) of two RGCs, the MI of bursting of two RGCs was calculated. Thus, for a pair of RGCs, the number of possible burst firing patterns is $2^{2}$, including the pattern $(0,0),(0,1),(1,0)$, and $(1,1)$. First, probability distribution of each firing pattern was obtained using the time interval of $100 \mathrm{~ms}$. Then, using the probability distributions of the four burst patterns, the MI was calculated using Eq. 3 .

For the control experiment, MI analysis was further performed assuming two simulated conditions of independent firing and perfectly synchronized firing between RGCs. First, to mimic the independent firing state, a surrogate burst train was created by shuffling the burst train of the reference cell (A). The shuffled train (A') was obtained by randomly rearranging the original burst train of the reference cell, destroying any relationship between the origin and the surrogate trains. Then, the MI between the reference cell and the surrogate cell $\left(A \leftrightarrow A^{\prime}\right)$ was calculated. Second, for perfectly synchronized firing, a duplicate train of the reference cell was created and MI analysis was performed $(\mathrm{A} \leftrightarrow \mathrm{A})$. Each of the shuffled condition and the perfectly synchronized condition represents the baseline and the upper bound of the mutual information between RGCs on pairwise condition. 


\section{Light stimulation}

We applied light stimulation to the retina to functionally classify RGCs and found the receptive fields (RFs) of RGCs. Briefly, we classified RGCs into two types, considering the spatiotemporal pattern of RGC RFs, where ON RGCs are considered as cells displaying a response when the flash is 'on', while OFF RGCs show a response when the flash is 'off'. Visual stimuli were generated by custom-made software, written in Matlab ${ }^{\mathrm{TM}}$ with Psychtoolbox $[27,28]$, and presented using a digital light processing (DLP) projector (ep7122; Hewlett-Packard, CA, USA) with a maximum resolution of $1,400 \times 1,050$ pixels and a refresh rate of $60 \mathrm{~Hz}$. The visual stimulus was projected onto the photoreceptor layer with a size of $1.7 \times 1.7 \mathrm{~mm}^{2}$ through the water dipping $20 \times$ objective lens (UMPLFLN20XW; Olympus Corp., Tokyo, Japan), with a beamsplitter (32-506; Edmund Optics Inc., NJ, USA) inserted into the light path for monitoring. Light stimuli were attenuated using a neutral density filter (NE220B; Thorlabs Inc., NJ, USA). For characterization of RFs of RGCs, we applied a spatiotemporal white noise stimulation, temporally updated at a frame rate of $30 \mathrm{~Hz}$, and spatially arranged in a checkerboard layout with a pixel width of $67 \mu \mathrm{m}$ (Fig. 1C). Each pixel was independently modulated according to the Bernoulli distribution with equal probabilities for +1 (white) and -1 (dark) (100\% contrast and mean intensity of 3.8 $\mu \mathrm{W} / \mathrm{cm}^{2}$ ). Light intensity corresponding to photopic vision was measured with a calibrated radiometer (ILT-5000; International Light Technologies, MA, USA). This white noise stimulation was applied for $15 \mathrm{~min}$.

\section{Spike-triggered average and receptive field estimation}

For an estimation of RGC RFs, the spatial maps of spiketriggered average (STA) were manipulated (Fig. 1C). Our previous study showed spatiotemporal patterns of mouse RFs through STA analysis [21]. Briefly, for STA, spikes from each RGC were counted to produce a train of spike counts binned at $33.3 \mathrm{~ms}(30 \mathrm{~Hz})$. The STA was obtained by averaging the stimuli preceding a spike with the time window of $400 \mathrm{~ms}$.

(Eq. 4)

$$
S T A(t)=\frac{1}{N} \sum_{n=1}^{N} s\left(t_{n}-t\right) w_{n}
$$

Here, $t_{n}$ is the time of the $n$th spike, $s\left(t_{n}-t\right)$ is the stimulus at the time preceding the spike time $t_{n}$ by $t, w_{n}$ is the number of spikes that occur in the time bin $t_{n}$ corresponding to the $n$th spike, and $N$ is the total number of spikes. First, temporal profiles of the STA were analyzed for each pixel. The spatial map of all the pixels at the temporal profile of maximum contrast was fit to a twodimensional Gaussian to parameterize the RGC RF. The mean and covariance of the fitted Gaussian distribution correspond to an ellipse. The RF diameter was defined as the geometric mean of the major and minor axes of the ellipse. The mean value was used as an indicator of the center of RGC.

(Eq. 5)

$R F$ diameter $=2 \sqrt{\text { major axes } \times \text { minor axes (of ellipse })}$

\section{Statistical analysis}

All results shown here, including burst parameters, BSI, and MI were represented by averaging for analyzed neurons. Their error bars were indicated with \pm standard error of the mean (SEM). In Fig. 3, the BSI was calculated for the inter-electrode distances. Their curves were plotted with the exponential fit. Significant difference between two species in a certain distance was verified with paired T-test $(*: \mathrm{p}<0.05, * *: \mathrm{p}<0.01, * * *: \mathrm{p}<0.001)$. To determine the statistical difference of BSIs among inter-electrode distances, ANOVA analysis $(\mathrm{p}<0.05)$ for each mouse and monkey retina was performed with post hoc tests of Turkey, Duncan, and Scheffe.

\section{RESULTS}

\section{Bursting activity in mouse and monkey retinas}

For burst analysis, we used 601 RGCs from 6 mouse retinas and 436 RGCs from 4 marmoset monkey retinas. First, we investigated the bursting patterns of RGCs from two species. Fig. 2A showed each representative burst from mouse and monkey retina. Compared with the mouse, the bursts of monkey RGCs showed remarkable bursting properties such as more spikes in a burst (Fig. 2A). Therefore, between mouse and monkey retinas, we compared burst parameters such as spike counts in a burst, inter-spike interval (ISI) in a burst, burst duration, burst frequency, and interburst interval (IBI) (Fig. 2B). For spike counts in a burst and burst frequency, monkey RGCs showed significantly more spike counts $(4.21 \pm 0.01)$ within a burst and burst frequency $(4.46 \pm 0.05 \mathrm{~Hz})$ compared with those of mouse RGCs $(3.67 \pm 0.01)$ and $(2.42 \pm 0.02$ $\mathrm{Hz}$ ), respectively. In monkey RGCs, averaged inter-spike interval in a burst (35.56 $\pm 0.29 \mathrm{~ms})$ and burst duration ( $114.20 \pm 1.56 \mathrm{~ms})$ are significantly shorter than in mouse RGCs (ISI: $51.67 \pm 0.26 \mathrm{~ms}$, burst duration: $138.40 \pm 1.36 \mathrm{~ms})$. For IBI, monkey RGCs showed shorter IBIs (222.97 $\pm 1.06 \mathrm{~ms})$ compared with mouse RGCs $(272.57 \pm 3.33 \mathrm{~ms})$. Overall, the bursts of monkey RGCs have more spikes over a short period than those of mouse RGCs, suggesting that compared with mouse RGC's bursts, monkey bursts could deliver retinal activity to the higher-order LGN neuron more reliably. 


\section{A. Representative spike trains of RGCs}

Monkey

Cell A

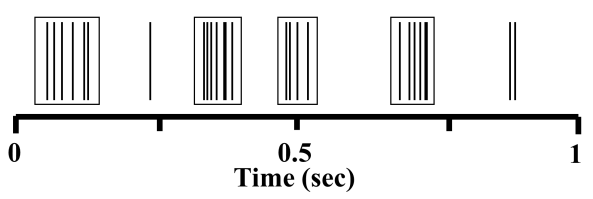

Cell B

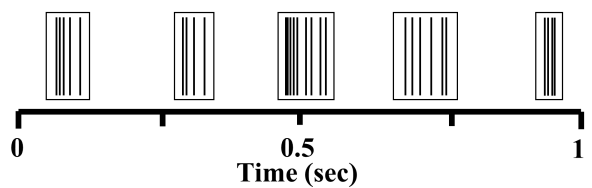

Cell A

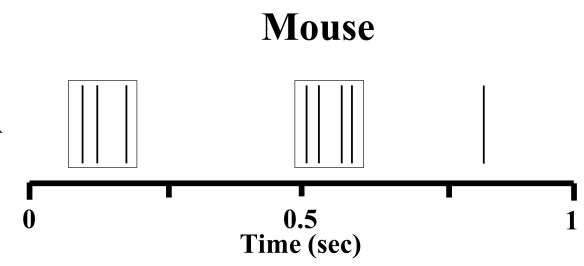

Cell B

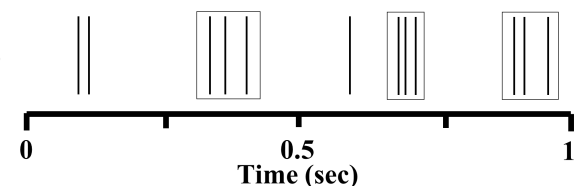

\section{B. Comparison of burst parameters between monkey and mouse RGCs}

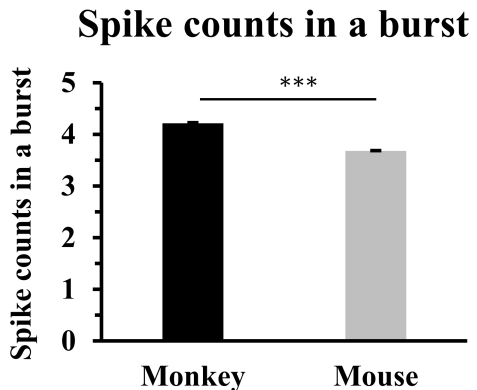

Burst frequency

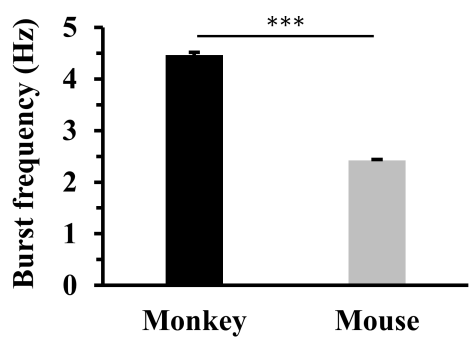

Inter-spike interval in a burst

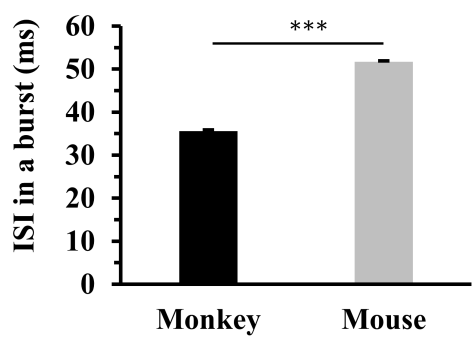

Inter-burst interval

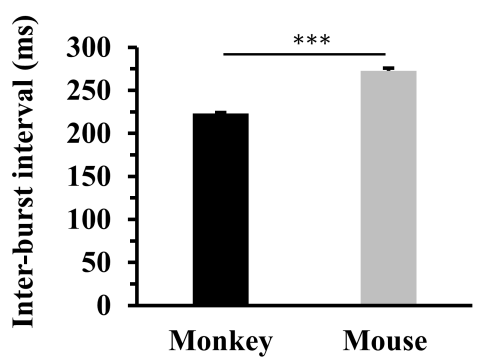

Fig. 2. Bursting activity in monkey and mouse retinas. (A) Representative spike trains of monkey and mouse RGCs. Raster plots show the burst firing of each RGC during one sec. Each solid line represents the timing of the spike. Square boxes indicate each burst of RGCs. (B) Comparison of burst parameters between monkey and mouse retinas: spike counts within a burst, inter-spike interval (ISI) within a burst, burst duration, burst frequency, and interburst interval (IBI). The statistical significance between the two species is shown as an asterisk $\left({ }^{* * *} \mathrm{p}<0.001\right)$.

Synchronized bursting patterns in monkey and mouse retinas

We investigated the synchronized bursting between RGC pairs from two species. Fig. 3A showed each representative RGC bursts in monkey and mouse retinas. Monkey RGCs showed a strong burst correlation between two RGCs compared with mouse RGCs. In the raster plot of two RGCs, the bursts of monkey RGCs were relatively well-synchronized in time. This synchronized pattern was well represented as a significant single peak through burst cross-correlogram, in which a single peak appeared within the time ranges of $100 \mathrm{~ms}$ from zero interval. On the other hand, mouse RGCs showed no correlated bursting between two RGCs, even with the nearest spacing of $200 \mu \mathrm{m}$. No significant peak in burst cross-correlogram suggests two RGCs have little correlated bursting.

To assess the strength of the burst correlation between RGC pairs, BSI was calculated for each RGC pair recorded on separate channels of MEA. For BSI calculation, we included only one RGC 


\section{A. Burst-correlation between the spike trains of two RGCs}
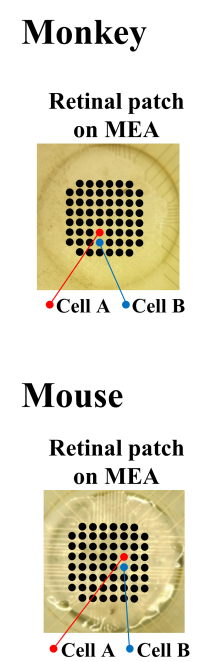

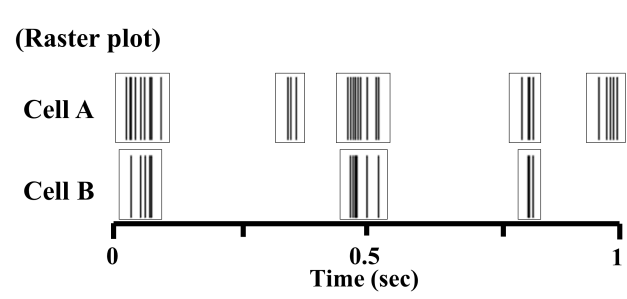

(Raster plot)

Cell A

Cell B

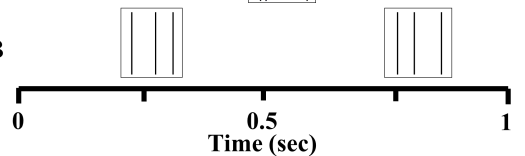

Burst cross-correlogram

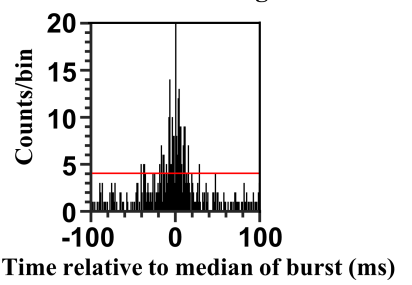

Burst cross-correlogram

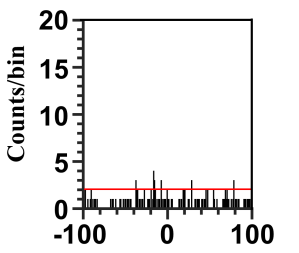

Time relative to median of burst (ms)

\section{B. Burst synchrony index as a function of distance $(\Delta t=100 \mathrm{~ms})$}

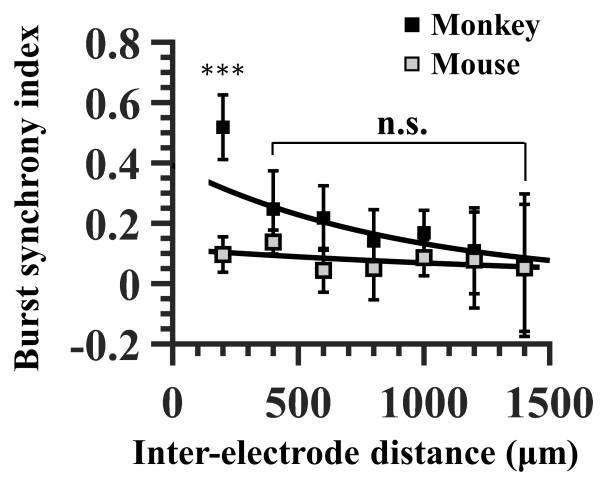

Fig. 3. Synchronized bursting patterns in monkey and mouse retinas. (A) Burst correlation between the spike trains of two RGCs. Left panel: the location of two RGCs with a spacing of $200 \mu \mathrm{m}$ onto MEA electrodes. Two pictures of the retinal patch on MEA are shown. Center panel: burst firing of two RGCs during one sec. Each solid line represents the timing of the spike. Square boxes indicate each burst of RGCs. Right panel: burst cross-correlogram. The time bins of the histograms are selected as $1 \mathrm{~ms}$. The red line indicates the significance level represented by the $99 \%$ confidence limit of the time histogram. (B) burst synchrony index (BSI) as a function of interelectrode distance. The time interval is chosen to be $100 \mathrm{~ms}$. The statistical difference between monkey and mouse when an inter-electrode distance between two RGCs is $200 \mu \mathrm{m}$ apart is shown as an asterisk $\left({ }^{* * *} \mathrm{p}<0.001\right)$. for each channel of MEA to exclude possible effects due to different RGC density or RF diameter across species. We used 193 RGCs (out of total 601 RGCs) from 6 mouse retinas and 156 RGCs (out of 436 RGCs) from 4 monkey retinas. More details are written in discussion section. Fig. 3B showed the BSI as a function of inter-electrode distance. A significant difference in BSI between two species appeared only for RGC pairs with $200 \mu \mathrm{m}$ spacing (***: $\mathrm{p}<0.001)$. The BSI of monkey RGCs decreased with the increment of the distance between RGC pairs, while mouse RGCs showed a near-zero BSIs regardless of distances between RGCs. Noticeably, the BSI of monkey RGC pairs with $200 \mu \mathrm{m}$ spacing was found to deviate from the fitting curve extrapolated only at the other distances.

To find out the statistical difference of BSIs among inter-electrode distances, we performed ANOVA analysis for mouse and monkey retina, respectively. In monkey retina, two statistically different groups (group 1: $200 \mu \mathrm{m}$, group 2: 400 1,400 $\mu \mathrm{m}, \mathrm{p}<0.05$ ) were found, while the mouse retina showed no significant differences along with the inter-electrode distances $(\mathrm{p}>0.05)$.

\section{Mutual information between RGCs across species}

How much information a pair of RGCs share through pairwise synchronized bursting? To answer this question, we performed MI analysis on synchronized bursting for a pair of RGCs. MI analysis was performed for RGC pairs with $200 \mu \mathrm{m}$ spacing, since a significant difference in BSI between two species appeared only for RGCs with $200 \mu \mathrm{m}$ apart (Fig. 3B).

As described in the method section for mutual information, we made shuffled train and perfect sync train. Each of the shuffledand the perfect conditions represents the baseline and the upper bound of the mutual information between RGCs on pairwise condition. The probability distribution of four possible firing patterns between two RGCs in shuffled, pairwise, and perfect sync train was observed (Fig. 4A). Across six histograms, the probability 


\section{A. Firing pattern distribution $\mathbf{P}(\mathbf{x})$}
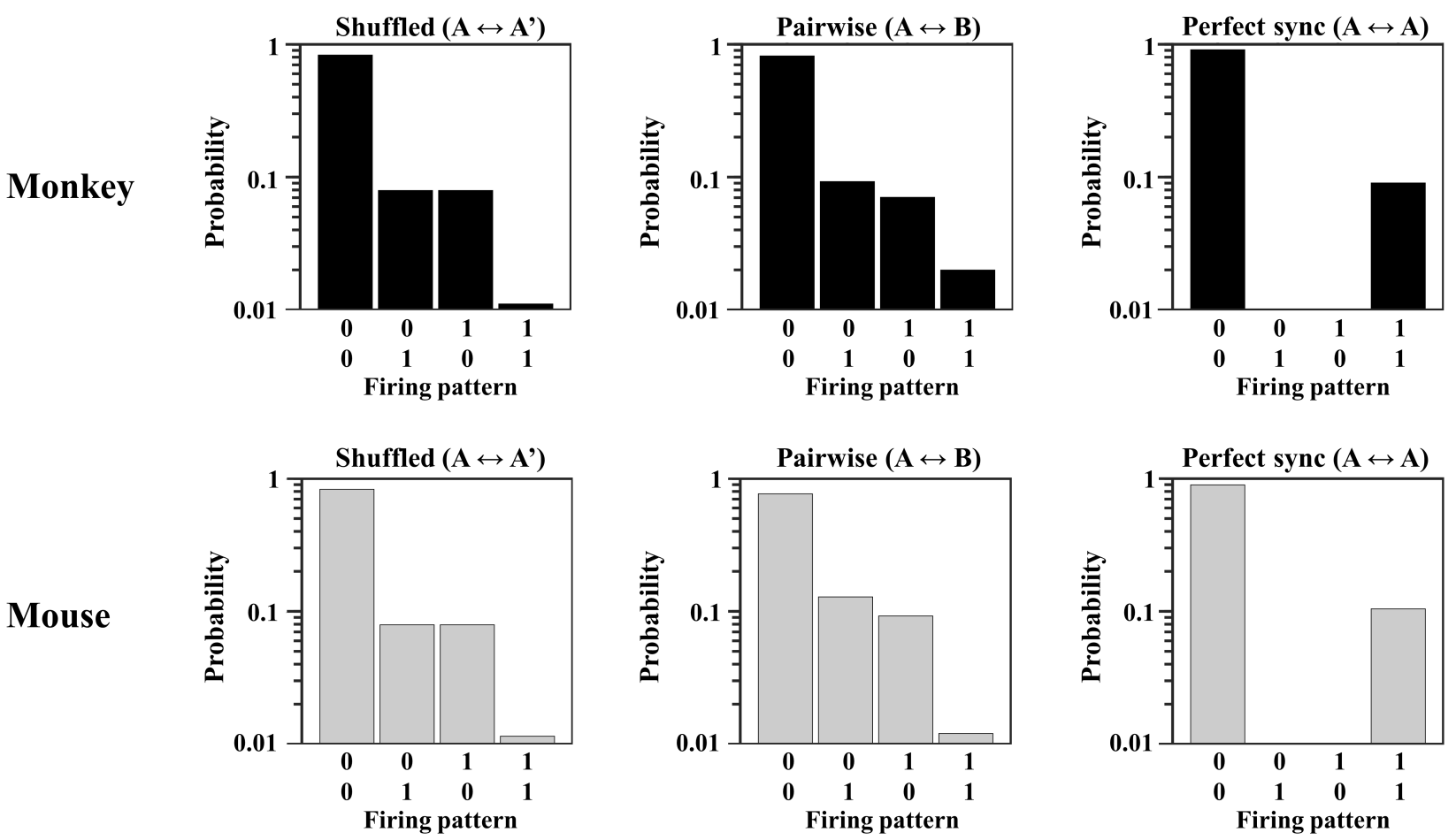

\section{B. Mutual information between RGCs}

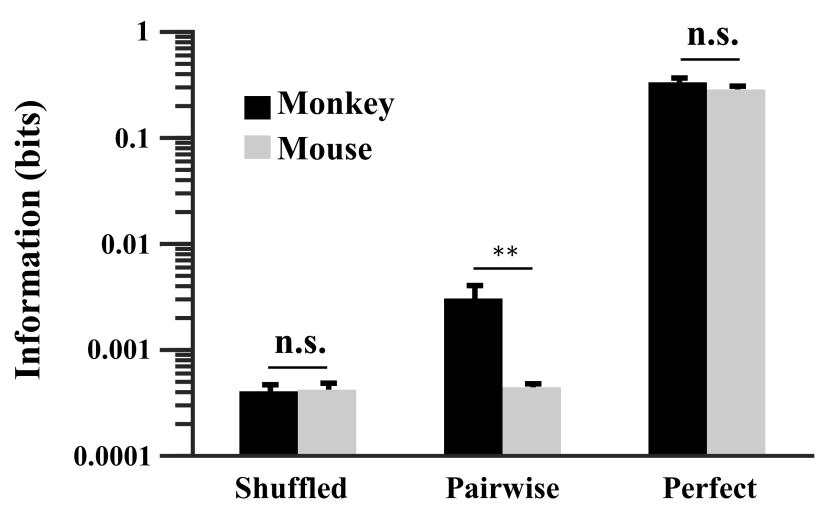

Fig. 4. Mutual information between RGCs in monkey and mouse. (A) Firing pattern distribution for pairwise RGCs with $200 \mu \mathrm{m}$ spacing between electrodes. Each distribution is represented by a histogram. The firing pattern is expressed in binary digits on the horizontal coordinate, indicating a burst (1) or no burst (0) in each cell. The probability of the vertical coordinate is noted on a logarithmic scale. Firing pattern distributions were obtained from shuffled data, pairwise data, and perfectly synchronized data. (B) Mutual information between a pair of RGCs. The statistical significance between the two species was shown as an asterisk $\left({ }^{*} \mathrm{p}<0.01\right)$. of $(0,0)$ firing pattern was always dominant. Also, each structure of the shuffled histogram and perfectly synchronized histogram is similar between monkey and mouse. The pairwise histogram of monkey RGCs showed an increase in probability for the $(1,1)$ pattern and a decrease in probability for the $(0,1),(1,0)$ patterns compared with the pairwise histogram of mouse RGCs. The pairwise histogram of mouse RGCs for the (1,1) pattern appears to be very similar to the shuffled histogram, which is the firing pattern distribution expected from independent firing. Based on these probability histograms, the following MIs were calculated respectively: For monkey, $I_{\text {(shuffled) }}=2.6 \times 10^{-4}, I_{\text {(pair })}=7.4 \times 10^{-3}, I_{\text {(perfect) }}=4.4 \times 10^{-1}$. For mouse, $I_{(\text {shuffled })}=1.0 \times 10^{-4}, I_{(\text {pair })}=4.7 \times 10^{-4}, I_{(\text {perfect })}=4.8 \times 10^{-1}$ bits.
For statistical analysis of MIs, 6726 pairs of monkey RGCs and 8482 pairs of mouse RGCs with $200 \mu \mathrm{m}$ spacing were used (Fig. 4B). Each group of the shuffled and perfect sync did not show any differences between two species $\left(I_{\text {(shuffled })}\right.$ : monkey $=4.1 \times 10^{-4}$, mouse $=4.2 \times 10^{-4}$ bits $/ I_{\text {(perfect) }}$ : monkey $=3.3 \times 10^{-1}$, mouse $=2.9 \times 10^{-1}$ bits). However, in the pairwise group, monkey's MI was found to be much higher than that of mouse $\left(I_{(p a i r)}\right.$ : monkey $=3.1 \times 10^{-3}$, mouse $=4.4 \times 10^{-4}$ bits). Given $I_{(\text {pair })} I_{\text {(shuffled })}$, through the pairwise burst correlation, each species of RGCs shares as much information as $2.7 \times 10^{-3}$ bits for monkey and $2.0 \times 10^{-5}$ bits for mouse, respectively. 
Spontaneous bursting activity for the ON and OFF RGC types

We showed a comparison of spontaneous bursting activity across species (Figs. 2, 3). However, a comparison of bursting activity for different physiological cell types was not shown. For this purpose, we performed a functional classification of RGCs using light stimulus. RGCs were classified into two types, $\mathrm{ON}$ and $\mathrm{OFF}$ RGCs (Detailed information can be found in the Method section, light stimulation and Spike-triggered average and receptive field estimation).

Out of six mouse retinas, $153 \mathrm{ON}$ - and $174 \mathrm{OFF}-\mathrm{RGCs}$ were harvested. Fig. $5 \mathrm{~A}$ shows the mosaics of receptive fields (RFs) of RGCs in a representative mouse retinal patch. Exemplary spatial and temporal RF profiles of RGCs are shown on the bottom side of the RF mosaic figures. Each spatial map of RFs was obtained from a specific recording channel (electrode number of MEA for ON cell in the mouse retina: 54, OFF cell in the mouse retina: 45). The ON RGCs shows a cluster of pixels with white color around the selected electrode, which has a positive contrast temporally. On the other hand, the OFF RGCs shows a negative contrast with black pixels. Each RF size of mouse ON and OFF RGCs was 259.0 \pm 63.9 $\mu \mathrm{m}$ and $276.6 \pm 67.0 \mu \mathrm{m}$, respectively. Additionally, there was no significant difference in RF size between mouse $\mathrm{ON}$ and $\mathrm{OFF}$ RGCs ( $\mathrm{p}>0.05)$.

Based on the classified ON and OFF RGCs, especially in the mouse retina, we investigated whether there is a difference in spontaneous bursting activity for each cell type. Fig. 5B and 5C show no significant difference between ON and OFF RGCs at all

\section{A. Mosaics of mouse RGC RFs}
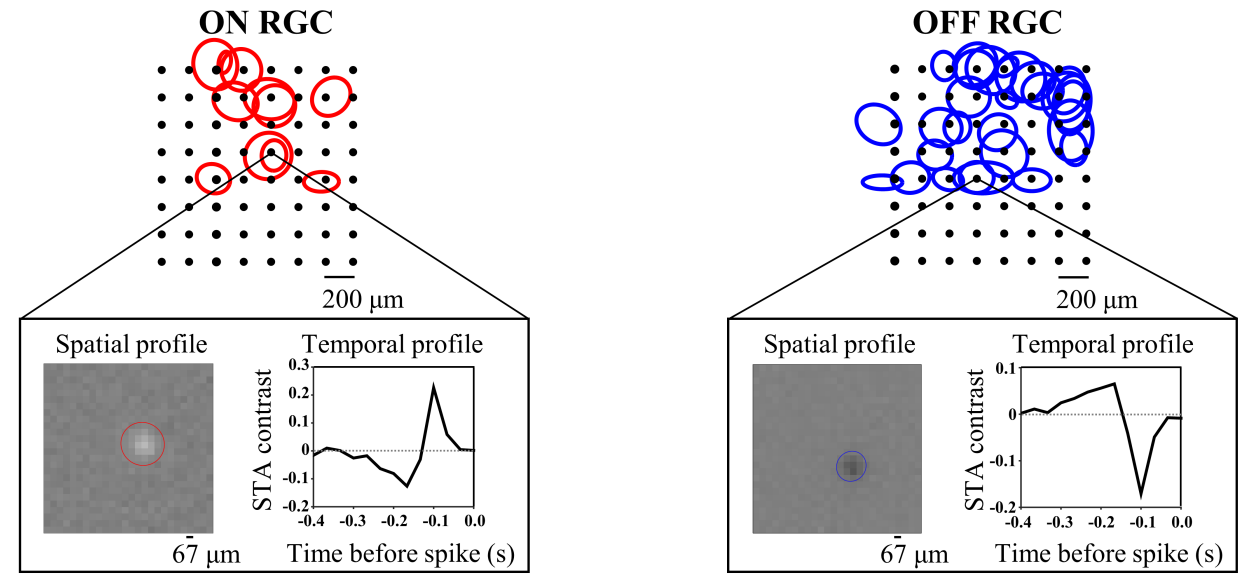

\section{B. Burst parameters}
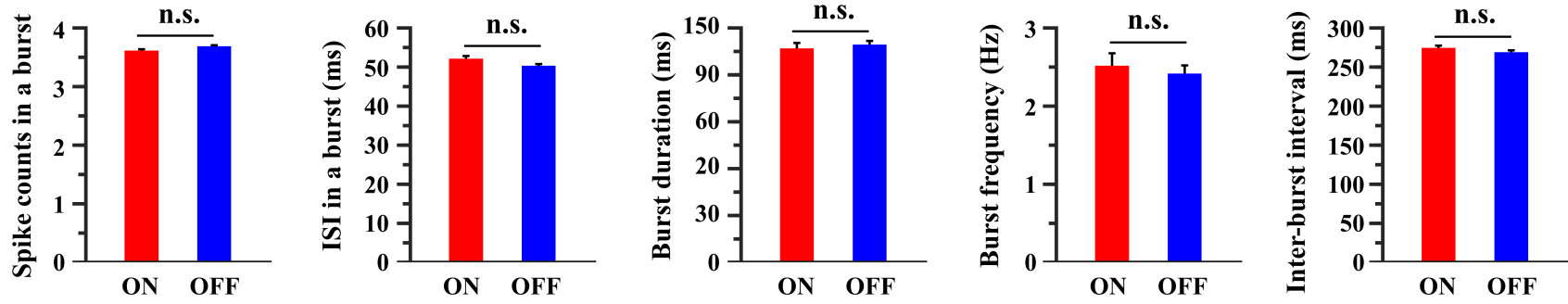

C. Burst synchronization

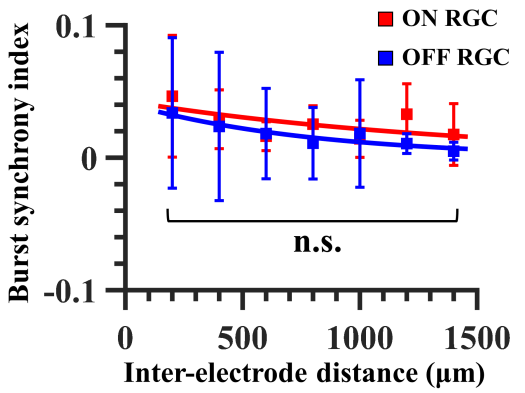

Fig. 5. Spontaneous bursting activity for the ON and OFF RGCs. (A) Top: mosaics of receptive fields (RFs) of RGCs reconstructed in each retinal patch from mouse are superimposed on the $8 \times 8$ electrodes; Bottom left: The spatial maps of STA from a selected electrode of MEA (electrode number of ON RGC in the mouse: 54, OFF RGC: 45). The RFs from spatial maps were fit to ellipse using two-dimensional Gaussian (ON RGC: red ellipse, OFF RGC: blue ellipse); Bottom right: The temporal profile of the maximum contrast pixel of $26 \times 26$ pixels. (B) Comparison of burst parameters between ON and OFF RGCs: spike counts within a burst, inter-spike interval (ISI) within a burst, burst duration, burst frequency, and inter-burst interval (IBI). (C) Burst synchrony index (BSI) as a function of interelectrode distance. There is no statistical difference between mouse ON and OFF RGCs. 
values, including bursting parameters and burst synchronization. It was impossible to compare the bursting activity between $\mathrm{ON}$ and OFF RGCs in monkeys since we only harvested three ON RGCs without any OFF RGCs out of monkey retinas. The difficulty of harvesting the light-sensitive RGCs in monkey retina will be mentioned in the discussion section.

\section{DISCUSSION}

\section{Bursting activity in mouse and monkey retinas}

In the nervous system, the coding scheme relates to how information is reliably transmitted through relays to target neurons. In the case of the visual system, it is associated with transmission through neural synaptic relays between RGCs and LGNs. Bursts are well known for their role in improving the reliability of information transfer (i.e., synaptic transmission) [3,4]. While a single spike may not pass through a synapse, multiple spikes in a burst will cross the synapse more reliably, improving the possibility of eliciting postsynaptic spikes. Besides, some studies reported that the correlation strength between neurons could be enhanced through the bursts rather than individual spikes $[29,30]$. The bursting activity can efficiently enhance the transfer of information either by storing information in the burst or by strengthening the connection with other neurons.

Our results showed that mouse RGCs are not preferential to bursting activity compared with marmoset monkey RGCs (Fig. 2). Given the low spike density, the importance of burst activity in the mouse retina is ambiguous. On the other hand, the burst statistics of monkey RGCs highlight that monkey RGCs exhibit higher spike counts in a burst. These findings indicate monkey RGCs transmit spike information much densely through burst carrier into the higher-order visual system than mouse RGCs do. Comprehensively, our results emphasize RGCs of mouse and monkey retinas have different bursting patterns, suggesting two species have distinctive retinal processing.

\section{Possible mechanisms of different bursting activity across species}

The main reasons for different bursting patterns between two species can be explained mainly by two factors, such as (1) Intrinsic RGC bursting created by intrinsic mechanism without any synaptic input [31], and (2) Input-driven bursting by presynaptic neurons of RGCs such as bipolar cells [32].

First, for intrinsic bursting, there are many discovered mechanisms of intrinsic bursting, including voltage-[33-35] and $\mathrm{Ca}^{2+}$ [36] gated ion channels. Typically, T-type voltage-gated calcium channel (T-type VGCC; transient opening calcium channels called $\left.\mathrm{Ca}_{\mathrm{v}} 3\right)$ is thought to be involved in the bursting process. Ttype VGCC allows the influx of calcium ions. Thus, T-type VGCC induces more frequent depolarization of membrane potential and causes high-frequency firing [37]. Due to its transient activation properties, T-type VGCC plays a role in the rhythmic bursts. In RGCs, T-type VGCC is known to be involved in the maintenance of intracellular $\mathrm{Ca}^{2+}$ concentration and the generation of RGC bursting [38]. T-type VGCC in RGCs has been identified in various species including the salamander [39], rat [31, 40, 41], turtle [42], and cat [43].

As another candidate channel for the intrinsic bursting process, $\mathrm{Ca}^{2+}$-activated $\mathrm{K}^{+}$channel $\left(\mathrm{K}_{\mathrm{Ca}}\right)$ has been reported to be essential for the modulation of bursting activity [44-46]. In particular, $\mathrm{K}_{\mathrm{Ca}}$ is involved in the control of bursts by affecting the hyperpolarization stage of the spike [47-50]. Activation of this channel leads to a decrease of sustained burst firing by increasing the spike threshold time. Blocking of $\mathrm{K}_{\mathrm{Ca}}$ with charybdotoxin results in burst firing by increasing the depolarization of the membrane potentials. Similarly, in RGCs, the $\mathrm{K}_{\mathrm{Ca}}$ channel contributes to the modulation of burst firing in RGCs [51], and blocking the $\mathrm{K}_{\mathrm{Ca}}$ channel increases the burst firing rate of RGCs [52]. These observations indicate that the modulation of $\mathrm{K}_{\mathrm{Ca}}$ is deeply involved in the spontaneous bursting of RGCs.

Comprehensively, the adjustment between excitation through T-type VGCCs and inhibition through $\mathrm{K}_{\mathrm{Ca}}$ leads to the intrinsic bursting of RGCs. The previous report in intact mouse retina showed that T-type VGCC amplitudes decreased from embryonic through postnatal stages [51]. Unfortunately, however, for the best knowledge of authors, there has been no study for the difference of expression levels of these channels according to the developmental stage on monkey RGC. However, we believe that the different channel density of these burst-associated channels in RGCs may provide a reasonable guess for understanding the distinct bursting activity between two species.

Second, for input-driven bursting by presynaptic neurons of RGC, a difference between rodent retina and primate retina has been reported recently [32]. Using immunohistochemistry in macaque and human retinas, Puthussery et al. showed that voltagegated sodium channel $\left(\mathrm{Na}_{\mathrm{V}} 1.1\right)$ known to be essential for the spike generation, was observed in an axon initial segment (AIS) region of bipolar cells. In other vertebrates, the $\mathrm{Na}_{V} 1.1$ was not seen in the AIS of bipolar cells. In rat and mouse retinas, $\mathrm{Na}_{\mathrm{V}} 1.1$ was found in AISs of the amacrine cell but not in bipolar cell axons. Thus, the high-density expression of $\mathrm{Na}_{\mathrm{V}} 1.1$ in bipolar cell axons seems to be a unique characteristic of the primate retina. Here, $\mathrm{Na}_{\mathrm{V}} 1.1$ is not a burst-associated ion channel, so it might not be directly related to the bursting activity of RGCs. However, it can be expected that 
different presynaptic inputs generated by channel specialization in bipolar cells will result in the different bursting activity of RGCs between two species.

\section{Comparison of synchronized burst firing between mouse and monkey retinas}

In this study, we showed comparisons of synchronized burst firing of RGCs in mouse and monkey retinas. For burst firing, we found that mouse RGCs showed little correlated firing, while monkey RGCs showed a strong correlation of bursts between RGC pairs.

Burst synchrony index (BSI), the strength of correlated firing between monkey RGCs decreased with the increment of the distance between RGC pairs. There are significant differences in BSI between mouse and monkey RGCs with a distance of $200 \mu \mathrm{m}$. In mouse retina, there is no significant difference in the synchronized bursting between RGC pairs irrespective of inter-electrode distances, even for the nearest spacing of $200 \mu \mathrm{m}$.

Since we used adult mice (>P56), our finding may not be generalized that mouse RGCs do not show the synchronized bursts throughout their lives. In fact, during the early development of visual circuits before eye-opening, mouse RGCs show strongly correlated bursts called retinal waves in the retina $[53,54]$. Their correlated activity works for the refinement of immature retinal circuits and the retinal projection between the retina and higherorder visual system, i.e., LGN and visual cortex. Burst correlation between RGCs appeared most strongly in early postnatal days (P9), became weakened during retinal development, and eventually reached to little synchronization at P42 close to adult age (P56) [53]. In conclusion, at infant age, the burst correlation contributes to the pruning of neural circuits, whereas it disappears at adult age. Thus, here in our study, as shown in Fig. 3, mice over P56 showed no correlated firing between a pair of RGCs and appeared to be independent bursts of each RGC. Unfortunately, for comparison, there have been no monkey studies related to burst correlation according to retinal development in our best knowledge. This issue is to be studied further in the future.

The reason why two species have different burst synchrony can be considered in two different aspects related to RGC: (1) Difference in RGC density, and (2) Different RF size of RGC. First, for RGC density, previous studies showed that the RGC density in primate fovea is higher than that of mouse RGCs [55-57]. The higher the RGC density, the shorter the average distance between RGCs. Second, for RF size, the average RF diameter of monkey RGCs is about $125 \mu \mathrm{m}$ in range of 50 to $200 \mu \mathrm{m}[58,59]$. In contrast, the average RF diameter of mouse RGCs is about $250 \mu \mathrm{m}$ ranging from 100 to $400 \mu \mathrm{m}$ [60-62], which mostly agrees with our result of Fig 5A (RF diameter of mouse ON and OFF RGCs: 259.0 \pm 63.9 $\mu \mathrm{m}$ and $276.6 \pm 67.0 \mu \mathrm{m})$. Thus, monkey RGCs showed smaller RF diameter than mouse RGCs on average. In our study, since we always compare the burst synchrony between two RGCs recorded on a separate channel of MEA, at least $200 \mu \mathrm{m}$ apart, the RF overlap may happen in the mouse, not the monkey retina. If there is RF overlap, it might increase the burst synchrony in the mouse, not the monkey retina. But we found higher burst synchrony in monkey retina. Therefore, we believe that the difference in burst synchrony is not due to different RGC density or RF diameter between the mouse and the monkey retina.

The other possibility for the inter-species difference in burst synchrony could be found in the different expression levels of gap junction channels. For spontaneous bursting, synchronized bursts are thought to be mainly generated through direct connections via a gap-junction channel called connexin-36 between RGCs [63]. The difference in the expression level of the gap junction channel between monkey and mouse would be one good candidate to explain the difference in synchronized bursts. But in our best knowledge, there has been no report for direct comparison of the expression level of the gap junction channel between mouse and monkey retina.

\section{Information: effect of pairwise burst correlation}

We found that while mouse RGCs have little information of $2.0 \times 10^{-5}$, monkey RGCs share around $2.7 \times 10^{-3}$ of information with one neighboring RGC through pairwise synchronized bursting. One can question the physiological meaning of such a burst correlation. At first glance, burst correlation may not seem to be efficient for transmission due to the critical problem of information redundancy. What are the benefits of burst correlation between neurons at the expense of redundancy?

Regarding population bursting, there can be two main benefits: (1) Compensation. Timely synchronized bursts would improve the reliability of information through shared information. During synaptic transmission, if one synapse fails to transmit information, the other synapse could serve as a complement. Thus, the success of information transmission would be guaranteed. (2) Amplification. The synchronized bursts would amplify the signal input by synaptic integration of multiple RGCs. It helps the EPSP in a postsynaptic of LGN to exceed the threshold potential, making it easier for LGN to fire output spike or burst. Besides, based on our knowledge that the RGC burst induces single synapse plasticity of LGN [17-19], we could assume that the synchronized population bursting of multiple synapses will result in more dynamic changes in synaptic plasticity than by a single synapse.

As a result, mouse RGCs are likely to transmit information in- 
dependently, suggesting that mouse RGCs select a coding scheme to minimize the redundancy of information. On the other hand, monkey RGCs seem to transmit information through mutual dependence. This finding suggests that the higher the evolutionary stage of the species, the more burst synchronization may be required for stable transmission and dynamic synaptic changes.

The next question is that how many RGCs are synchronized, that is, what is the optimal structure of the primate retinal network to satisfy both reliable information transmission and low redundancy. Greater synchrony between RGCs would enhance reliable information transmission to the LGN. However, if the synchrony between RGCs is too high, the amount of transmitted information would be lower. In future works, we would like to investigate more complex networks (i.e., burst correlation among three or more neurons). By this, we could answer how many neurons are needed to maximize mutual information, which will improve our understanding of the functional role of the population bursting for information transmission.

\section{Constraint on harvesting the light-sensitive RGCs in the monkey retina}

Harvesting light-sensitive RGCs in monkey retina was problematic due to some constraints. It usually takes at least about 40 minutes to transport monkey eyeballs after enucleation from OSONG KBIO HEALTH to our lab. During the retinal preparation, we could often observe the retinal pigment epithelium (RPE) layer, supporting essential nutrients to the retina, was detached severely from the retina. RPE detachment from the retina can induce severe damage to the nearest photoreceptors (PRs) rather than the relatively distant RGCs. In some experiments of RPE detachment, after removal of RPE, the visual pathway from PR to RGC was functionally inactive while RGC's spontaneous firing was well maintained and robust [64-66]. To improve the tissue quality during the time window from enucleation to recording, we used a $\mathrm{CO}_{2}$-independent medium well known as transport buffer to avoid tissue damage caused by $\mathrm{pH}$ changes of the buffer solution. However, despite these efforts, there was substantial tissue damage. A fundamental modification of the experimental procedure is required, such as shorter transfer time, to harvest light-responsive RGCs reliably.

\section{Future work}

\section{The bursting activity of light-stimulated RGCs}

Here, we investigated the bursting patterns and burst synchronization of RGC bursts without light stimulus across species. As a next step, we would like to examine the bursting activity of light-stimulated RGCs across species. Many studies address that the burst can code different stimulus features and expand the additional coding space using the number of spikes and temporal patterns (i.e., ISI) within a burst [67-70]. Recently, some studies showed that thalamocortical relay (TCR) neurons or pyramidal neurons use a multiplexed neural code, which distinguishes multiple input streams through burst parameters, including the burst duration and ISI within a burst $[71,72]$. Accordingly, burst parameters, including the number of spikes and ISIs in a burst, and burst duration, etc., could be employed to discriminate the multiple stimulus features about what and when of the stimuli.

In this context, with a visual stimulus, the bursting activity may differ from spontaneous bursting. For the additional coding space, some burst parameters, including spike count and burst duration, can increase further compared with spontaneous bursting. Besides, the increase of burst duration may induce the increase of IBI for full recovery during the quiescence period before the neuron can burst again.

It will be essential to investigate the change in burst synchronization with a visual stimulus. For spontaneous bursting, synchronized bursts are mainly generated through direct connections by a gap-junction channel called connexin-36 between RGCs [63]. With a visual stimulus, the light activates a series of retinal circuits, including photoreceptors and bipolar cells. Then, light-driven presynaptic inputs induce RGC firing. Network-mediated input through a chemical synapse by a bipolar cell relay to RGCs will further increase burst synchronization. This network-mediated input to RGCs may augment the burst correlation in mouse retina as well as in monkey retina. It would be interesting to observe if the species difference in the burst synchrony index will be preserved even with visual stimuli.

\section{Need for in vivo dual recording of RGC and LGN}

Our study showed an in-vitro assessment of RGC population activity using a multi-channel recording system. Since the simultaneous recording of multiple RGCs was possible, timely synchronized bursts could be found in the retina, especially in monkey RGCs with a spacing of $200 \mu \mathrm{m}$. In conclusion, we suggest that the pairwise burst correlation between RGCs contributes to reliable information transfer to LGN.

Our future work is to investigate how correlated bursting activity contributes to the changes in LGN. To do this, we must record RGC and LGN signals simultaneously. Some studies showed that in vivo dual recording experiments were performed on single-unit recordings of RGC and LGN [17, 73]. For RGC recording, they inserted a single parylene-coated tungsten microelectrode into the eye with the aid of an intraocular guide tube and a custommade manipulator. For LGN recording, a series of surgical proce- 
dures such as craniotomy were performed. Subsequently, a single electrode was placed into the LGN, considering the retinotopic position of the LGN associated with the recorded RGC. A flexible multi-electrode chip is attached well to the RGC layer to record population activity of retinogeniculate neurons [74]. For LGN recordings, a high-density depth probe into the LGN [75] could be useful tools. Through this in vivo study, it will be possible to better understand the role of the RGC network for information transfer to LGN in the primate retinogeniculate system, which eventually helps us to comprehend the general strategy for information transmission through multiple communication in the nervous system.

\section{ACKNOWLEDGEMENTS}

We thank the Laboratory Animal Center of the OSONG KBIO HEALTH for providing us the marmoset monkey eyeballs. This research was supported in part by the Bio \& Medical Technology Development Program (NRF-2017M3A9E2056460 \& 2017M3A9E2062707), the Basic Science Research Program (NRF2018R1A2B6003917) through the National Research Foundation of Korea (NRF) funded by the Ministry of Science and ICT (MSIP), and an Incheon National University Research Grant in 2017. This work was financially supported by the research year of Chungbuk National University in 2018, in part.

\section{REFERENCES}

1. Johnson KO (2000) Neural coding. Neuron 26:563-566.

2. Gerstner W, Kistler WM (2002) Spiking neuron models: single neurons, populations, plasticity. pp xiv, 480. Cambridge University Press, Cambridge, CA.

3. Krahe R, Gabbiani F (2004) Burst firing in sensory systems. Nat Rev Neurosci 5:13-23.

4. Snider RK, Kabara JF, Roig BR, Bonds AB (1998) Burst firing and modulation of functional connectivity in cat striate cortex. J Neurophysiol 80:730-744.

5. Dobrunz LE, Stevens CF (1997) Heterogeneity of release probability, facilitation, and depletion at central synapses. Neuron 18:995-1008.

6. Rosenmund C, Clements JD, Westbrook GL (1993) Nonuniform probability of glutamate release at a hippocampal synapse. Science 262:754-757.

7. Lisman JE (1997) Bursts as a unit of neural information: making unreliable synapses reliable. Trends Neurosci 20:38-43.

8. Sengupta M, Thirumalai V (2015) AMPA receptor mediated synaptic excitation drives state-dependent bursting in Purkinje neurons of zebrafish larvae. Elife 4:e09158.
9. Casado M, Dieudonné S, Ascher P (2000) Presynaptic Nmethyl-D-aspartate receptors at the parallel fiber-Purkinje cell synapse. Proc Natl Acad Sci U S A 97:11593-11597.

10. Sherman SM (2001) Tonic and burst firing: dual modes of thalamocortical relay. Trends Neurosci 24:122-126.

11. Jahnsen H, Llinás R (1984) Ionic basis for the electro-responsiveness and oscillatory properties of guinea-pig thalamic neurones in vitro. J Physiol 349:227-247.

12. Shai AS, Anastassiou CA, Larkum ME, Koch C (2015) Physiology of layer 5 pyramidal neurons in mouse primary visual cortex: coincidence detection through bursting. PLoS Comput Biol 11:e1004090.

13. Thomas MJ, Watabe AM, Moody TD, Makhinson M, O'Dell TJ (1998) Postsynaptic complex spike bursting enables the induction of LTP by theta frequency synaptic stimulation. J Neurosci 18:7118-7126.

14. Gjorgiieva J, Toyoizumi T, Eglen SJ (2009) Burst-time-dependent plasticity robustly guides ON/OFF segregation in the lateral geniculate nucleus. PLoS Comput Biol 5:e1000618.

15. Gollisch T, Meister M (2010) Eye smarter than scientists believed: neural computations in circuits of the retina. Neuron 65:150-164.

16. Wong RO, Oakley DM (1996) Changing patterns of spontaneous bursting activity of on and off retinal ganglion cells during development. Neuron 16:1087-1095.

17. Alitto H, Rathbun DL, Vandeleest JJ, Alexander PC, Usrey WM (2019) The augmentation of retinogeniculate communication during thalamic burst mode. J Neurosci 39:56975710 .

18. Litvina EY, Chen C (2017) An evolving view of retinogeniculate transmission. Vis Neurosci 34:E013.

19. Moore BD 4th, Kiley CW, Sun C, Usrey WM (2011) Rapid plasticity of visual responses in the adult lateral geniculate nucleus. Neuron 71:812-819.

20. Ahn KN, Ahn JY, Kim JH, Cho K, Koo KI, Senok SS, Goo YS (2015) Effect of stimulus waveform of biphasic current pulse on retinal ganglion cell responses in retinal degeneration $(\mathrm{rd} 1)$ mice. Korean J Physiol Pharmacol 19:167-175.

21. Ahn J, Rueckauer B, Yoo Y, Goo YS (2020) New features of receptive fields in mouse retina through spike-triggered covariance. Exp Neurobiol 29:38-49.

22. Reinhard K, Tikidji-Hamburyan A, Seitter H, Idrees S, Mutter M, Benkner B, Münch TA (2014) Step-by-step instructions for retina recordings with perforated multi electrode arrays. PLoS One 9:e106148.

23. Lewicki MS (1998) A review of methods for spike sorting: the detection and classification of neural action potentials. Net- 
work 9:R53-R78.

24. Stafford BK, Sher A, Litke AM, Feldheim DA (2009) Spatialtemporal patterns of retinal waves underlying activity-dependent refinement of retinofugal projections. Neuron 64:200212.

25. Legéndy CR, Salcman M (1985) Bursts and recurrences of bursts in the spike trains of spontaneously active striate cortex neurons. J Neurophysiol 53:926-939.

26. Shlens J, Field GD, Gauthier JL, Grivich MI, Petrusca D, Sher A, Litke AM, Chichilnisky EJ (2006) The structure of multineuron firing patterns in primate retina. J Neurosci 26:82548266.

27. Brainard DH (1997) The psychophysics toolbox. Spat Vis 10:433-436.

28. Pelli DG (1997) The VideoToolbox software for visual psychophysics: transforming numbers into movies. Spat Vis 10:437-442.

29. Chan HK, Yang DP, Zhou C, Nowotny T (2016) Burst firing enhances neural output correlation. Front Comput Neurosci 10:42.

30. Kepecs A, Lisman J (2003) Information encoding and computation with spikes and bursts. Network 14:103-118.

31. Sargoy A, Sun X, Barnes S, Brecha NC (2014) Differential calcium signaling mediated by voltage-gated calcium channels in rat retinal ganglion cells and their unmyelinated axons. PLoS One 9:e84507.

32. Puthussery T, Venkataramani S, Gayet-Primo J, Smith RG, Taylor WR (2013) NaV1.1 channels in axon initial segments of bipolar cells augment input to magnocellular visual pathways in the primate retina. J Neurosci 33:16045-16059.

33. Butera RJ Jr, Rinzel J, Smith JC (1999) Models of respiratory rhythm generation in the pre-Bötzinger complex. I. bursting pacemaker neurons. J Neurophysiol 82:382-397.

34. Huguenard JR, McCormick DA (1992) Simulation of the currents involved in rhythmic oscillations in thalamic relay neurons. J Neurophysiol 68:1373-1383.

35. Wang XJ (1999) Fast burst firing and short-term synaptic plasticity: a model of neocortical chattering neurons. Neuroscience 89:347-362.

36. Kloppenburg P, Zipfel WR, Webb WW, Harris-Warrick RM (2000) Highly localized $\mathrm{Ca}(2+)$ accumulation revealed by multiphoton microscopy in an identified motoneuron and its modulation by dopamine. J Neurosci 20:2523-2533.

37. Catterall WA (2011) Voltage-gated calcium channels. Cold Spring Harb Perspect Biol 3:a003947.

38. Lee SC, Hayashida Y, Ishida AT (2003) Availability of lowthreshold Ca2+ current in retinal ganglion cells. J Neuro- physiol 90:3888-3901.

39. Henderson D, Miller RF (2003) Evidence for low-voltageactivated (LVA) calcium currents in the dendrites of tiger salamander retinal ganglion cells. Vis Neurosci 20:141-152.

40. Guenther E, Rothe T, Taschenberger H, Grantyn R (1994) Separation of calcium currents in retinal ganglion cells from postnatal rat. Brain Res 633:223-235.

41. Karschin A, Lipton SA (1989) Calcium channels in solitary retinal ganglion cells from post-natal rat. J Physiol 418:379396.

42. Liu Y, Lasater EM (1994) Calcium currents in turtle retinal ganglion cells. I. the properties of T- and L-type currents. J Neurophysiol 71:733-742.

43. Huang SJ, Robinson DW (1998) Activation and inactivation properties of voltage-gated calcium currents in developing cat retinal ganglion cells. Neuroscience 85:239-247.

44. Nemargut JP, Zhu J, Savoie BT, Wang GY (2009) Differential effects of charybdotoxin on the activity of retinal ganglion cells in the dark- and light-adapted mouse retina. Vision Res 49:388-397.

45. Wang GY, Robinson DW, Chalupa LM (1998) Calciumactivated potassium conductances in retinal ganglion cells of the ferret. J Neurophysiol 79:151-158.s

46. Zhong YS, Wang J, Liu WM, Zhu YH (2013) Potassium ion channels in retinal ganglion cells (review). Mol Med Rep 8:311-319.

47. Constanti A, Sim JA (1987) Calcium-dependent potassium conductance in guinea-pig olfactory cortex neurones in vitro. J Physiol 387:173-194.

48. Lancaster B, Pennefather P (1987) Potassium currents evoked by brief depolarizations in bull-frog sympathetic ganglion cells. J Physiol 387:519-548.

49. Schwindt PC, Spain WJ, Foehring RC, Stafstrom CE, Chubb MC, Crill WE (1988) Multiple potassium conductances and their functions in neurons from cat sensorimotor cortex in vitro. J Neurophysiol 59:424-449.

50. Adams PR, Constanti A, Brown DA, Clark RB (1982) Intracellular Ca2+ activates a fast voltage-sensitive $\mathrm{K}+$ current in vertebrate sympathetic neurones. Nature 296:746-749.

51. Rothe T, Jüttner R, Bähring R, Grantyn R (1999) Ion conductances related to development of repetitive firing in mouse retinal ganglion neurons in situ. J Neurobiol 38:191-206.

52. Frings S, Brüll N, Dzeja C, Angele A, Hagen V, Kaupp UB, Baumann A (1998) Characterization of ether-à-go-go channels present in photoreceptors reveals similarity to $\mathrm{IKx}$, a K+ current in rod inner segments. J Gen Physiol 111:583-599.

53. Demas J, Eglen SJ, Wong RO (2003) Developmental loss of 
synchronous spontaneous activity in the mouse retina is independent of visual experience. J Neurosci 23:2851-2860.

54. Xu HP, Burbridge TJ, Ye M, Chen M, Ge X, Zhou ZJ, Crair MC (2016) Retinal wave patterns are governed by mutual excitation among starburst amacrine cells and drive the refinement and maintenance of visual circuits. J Neurosci 36:3871-3886.

55. Bleckert A, Schwartz GW, Turner MH, Rieke F, Wong RO (2014) Visual space is represented by nonmatching topographies of distinct mouse retinal ganglion cell types. Curr Biol 24:310-315.

56. Jang J, Song M, Paik SB (2020) Retino-cortical mapping ratio predicts columnar and salt-and-pepper organization in mammalian visual cortex. Cell Rep 30:3270-3279.e3.

57. Vidne M, Ahmadian Y, Shlens J, Pillow JW, Kulkarni J, Litke AM, Chichilnisky EJ, Simoncelli E, Paninski L (2012) Modeling the impact of common noise inputs on the network activity of retinal ganglion cells. J Comput Neurosci 33:97-121.

58. Chichilnisky EJ, Kalmar RS (2002) Functional asymmetries in $\mathrm{ON}$ and OFF ganglion cells of primate retina. J Neurosci 22:2737-2747.

59. Rhoades CE, Shah NP, Manookin MB, Brackbill N, Kling A, Goetz G, Sher A, Litke AM, Chichilnisky EJ (2019) Unusual physiological properties of smooth monostratified ganglion cell types in primate retina. Neuron 103:658-672.e6.

60. Baden T, Berens P, Franke K, Román Rosón M, Bethge M, Euler T (2016) The functional diversity of retinal ganglion cells in the mouse. Nature 529:345-350.

61. Jouty J, Hilgen G, Sernagor E, Hennig MH (2018) Non-parametric physiological classification of retinal ganglion cells in the mouse retina. Front Cell Neurosci 12:481.

62. Pillow JW, Shlens J, Paninski L, Sher A, Litke AM, Chichilnisky EJ, Simoncelli EP (2008) Spatio-temporal correlations and visual signalling in a complete neuronal population. Nature 454:995-999.

63. Trenholm S, Awatramani GB (1995) Myriad roles for gap junctions in retinal circuits. In: Webvision: the organization of the retina and visual system (Kolb H, Fernandez E, Nelson R, eds). University of Utah Health Sciences Center, Salt Lake City, UT.
64. Alarautalahti V, Ragauskas S, Hakkarainen JJ, Uusitalo-Järvinen H, Uusitalo H, Hyttinen J, Kalesnykas G, Nymark S (2019) Viability of mouse retinal explant cultures assessed by preservation of functionality and morphology. Invest Ophthalmol Vis Sci 60:1914-1927.

65. Lo AC, Woo TT, Wong RL, Wong D (2011) Apoptosis and other cell death mechanisms after retinal detachment: implications for photoreceptor rescue. Ophthalmologica 226 Suppl 1:10-17.

66. Murakami Y, Notomi S, Hisatomi T, Nakazawa T, Ishibashi T, Miller JW, Vavvas DG (2013) Photoreceptor cell death and rescue in retinal detachment and degenerations. Prog Retin Eye Res 37:114-140.

67. Zeldenrust F, Chameau P, Wadman WJ (2018) Spike and burst coding in thalamocortical relay cells. PLoS Comput Biol 14:e1005960.

68. Eyherabide HG, Rokem A, Herz AV, Samengo I (2009) Bursts generate a non-reducible spike-pattern code. Front Neurosci 3:8-14.

69. Kepecs A, Wang XJ, Lisman J (2002) Bursting neurons signal input slope. J Neurosci 22:9053-9062.

70. Reinagel P, Reid RC (2000) Temporal coding of visual information in the thalamus. J Neurosci 20:5392-5400.

71. Mease RA, Kuner T, Fairhall AL, Groh A (2017) Multiplexed spike coding and adaptation in the thalamus. Cell Rep 19:1130-1140.

72. Naud R, Sprekeler H (2018) Sparse bursts optimize information transmission in a multiplexed neural code. Proc Natl Acad Sci U S A 115:E6329-E6338.

73. Suematsu N, Naito T, Miyoshi T, Sawai H, Sato H (2013) Spatiotemporal receptive field structures in retinogeniculate connections of cat. Front Syst Neurosci 7:103.

74. Winkin N, Mokwa W (2012) Flexible multi-electrode array with integrated bendable CMOS-chip for implantable systems. Conf Proc IEEE Eng Med Biol Soc 2012:3882-3885.

75. Jia X, Siegle JH, Bennett C, Gale SD, Denman DJ, Koch C, Olsen SR (2019) High-density extracellular probes reveal dendritic backpropagation and facilitate neuron classification. J Neurophysiol 121:1831-1847. 